\title{
Another proof of Pell identities by using the determinant of tridiagonal matrix
}

\author{
Meral Yaşar*\&Durmuş Bozkurt ${ }^{\dagger}$ \\ Department of Mathematics, Nigde University and \\ Department of Mathematics, Selcuk University
}

May 25, 2018

\begin{abstract}
In this paper, another proof of Pell identities is presented by using the determinant of tridiagonal matrices. It is calculated via the Laplace expansion.
\end{abstract}

Key words: Pell numbers, Pell identities, tridiagonal matrix, Laplace expansion, determinant.

\section{Introduction}

Pell numbers are defined as

$$
P_{n}=2 P_{n-1}+P_{n-2}
$$

with the initial conditions $P_{0}=0, P_{1}=1$ for $n \geq 2$.

In [1, a complex factorization formula for $(n+1) t h$ Pell number is obtained to provided the tridiagonal matrix

$$
N(n)=\left[\begin{array}{cccccc}
2 i & 1 & & & & \\
1 & 2 i & 1 & & & \\
& 1 & 2 i & 1 & & \\
& & \ddots & \ddots & \ddots & \\
& & & 1 & 2 i & 1 \\
& & & & 1 & 2 i
\end{array}\right]
$$

as in the following:

$$
P_{n+1}=m|N(n)|, \quad m=\left\{\begin{array}{rr}
1, & n \equiv 0(\bmod 4) \\
-i, & n \equiv 1(\bmod 4) \\
-1, & n \equiv 2(\bmod 4) \\
i, & n \equiv 3(\bmod 4)
\end{array} .\right.
$$

*e-mail:myasar@nigde.edu.tr

†e-mail:dbozkurt@selcuk.edu.tr 
In [2], an identity of Fibonacci numbers is proved via the determinant of the tridiagonal matrix. In [3], the authors showed the connection between Fibonacci numbers and Chebyshev polynomials and obtained a complex factorization for Fibonacci numbers by using a sequence of the tridiagonal matrices. Then with a small difference in the tridiagonal matrix, it is showed that how Lucas numbers and Chebyshev polynomials are connected to each other. Two complex factorization are obtained by using the $n \times n$ tridiagonal and anti-tridiagonal matrix for $n$ is even in [4.

In this paper, we give another proof of Pell identities

$$
\begin{gathered}
P_{2 n}=P_{n}\left(P_{n+1}+P_{n-1}\right) \\
P_{n}=P_{k} P_{n-k+1}+P_{k-1} P_{n-k}
\end{gathered}
$$

where $k$ is a positive integer. For $1 \leq k \leq n$

$$
\begin{gathered}
P_{n}=P_{1} P_{n}+P_{0} P_{n-1} \\
P_{n}=P_{2} P_{n-1}+P_{1} P_{n-2} \\
P_{n}=P_{3} P_{n-2}+P_{2} P_{n-3} \\
P_{n}=P_{4} P_{n-3}+P_{3} P_{n-4} \\
\vdots \\
P_{n}=P_{n} P_{1}+P_{n-1} P_{0} .
\end{gathered}
$$

\section{Main Result}

Let $A$ be an $n \times n$ matrix, $A\left(\left[i_{1}, i_{2}, \ldots, i_{k}\right],\left[j_{1}, j_{2}, \ldots, j_{k}\right]\right)$ be the $k \times k$ submatrix of $A$ and $M\left(\left[i_{1}, i_{2}, \ldots, i_{k}\right],\left[j_{1}, j_{2}, \ldots, j_{k}\right]\right)$ be the $(n-k) \times(n-k)$ minor of the matrix $A$. The cofactor of $A$ is defined by

$$
\AA\left(\left[i_{1}, i_{2}, \ldots, i_{k}\right],\left[j_{1}, j_{2}, \ldots, j_{k}\right]\right)=(-1)^{m} M\left(\left[i_{1}, i_{2}, \ldots, i_{k}\right],\left[j_{1}, j_{2}, \ldots, j_{k}\right]\right)
$$

where $1 \leq i_{1}, i_{2}, \cdots, i_{k} \leq n$ and $m=\sum_{r=1}^{k}\left(i_{r}+j_{r}\right)$.

The determinant of the matrix $A$ is

$$
\begin{aligned}
\operatorname{det}(A)= & \sum_{\substack{1 \leq i_{1}, i_{2}, \cdots, i_{k} \leq n\\
}} \operatorname{det}\left(A\left(\left[i_{1}, i_{2}, \ldots, i_{k}\right],\left[j_{1}, j_{2}, \ldots, j_{k}\right]\right)\right) \\
& \times \operatorname{det}\left(\AA\left(\left[i_{1}, i_{2}, \ldots, i_{k}\right],\left[j_{1}, j_{2}, \ldots, j_{k}\right]\right)\right) .
\end{aligned}
$$

If $A(i, j)=a_{i j}$, then $\AA(i, j)=(-1)^{i+j} M(i, j)=\AA_{i j}$ and the determinant is

$$
\operatorname{det}(A)=\sum_{i=1}^{n} a_{i j} \stackrel{\AA}{i j}_{i j}
$$

This is the famous Laplace expansion formula 2]. We will use this formula to proof of Pell identities in (3). 
The cofactors of the first row of the matrix $N(n)$ are

$$
\begin{gathered}
\stackrel{\circ}{11}_{11}=\left\{\begin{aligned}
-P_{n-1} & , n \equiv 0(\bmod 4) \\
-i P_{n-1} & , n \equiv 1(\bmod 4) \\
P_{n-1} & , n \equiv 2(\bmod 4) \\
i P_{n-1} & , n \equiv 3(\bmod 4)
\end{aligned}\right. \\
\stackrel{A}{12}_{12}=\left\{\begin{array}{rl}
-i P_{n-2} & , n \equiv 0(\bmod 4) \\
P_{n-2} & , n \equiv 1(\bmod 4) \\
i P_{n-2} & , n \equiv 2(\bmod 4) \\
-P_{n-2} & , n \equiv 3(\bmod 4)
\end{array} .\right.
\end{gathered}
$$

By using the Laplace expansion formula the determinant of the matrix $N(n-1)$ is

$$
\operatorname{det}(N(n-1))=2 P_{n-1}+P_{n-2} .
$$

From (1), the initial value $P_{0}=0, P_{1}=1, P_{2}=2$ and the fact $P_{2}=2 P_{1}$ are used in (5), then we have

$$
\begin{aligned}
P_{n} & =2 P_{1} P_{n-1}+P_{1} P_{n-2} \\
& =P_{2} P_{n-1}+P_{1} P_{n-2} .
\end{aligned}
$$

If the first two rows of the matrix $N(n-1)$ are chosen, there are only three $2 \times 2$ submatrices of the matrix $N(n-1)$ whose determinants are nonzero. i.e.

$$
\begin{aligned}
& A([1,2],[1,2])=\left[\begin{array}{cc}
2 i & 1 \\
1 & 2 i
\end{array}\right]=-P_{3} \\
& A([1,2],[1,3])=\left[\begin{array}{cc}
2 i & 0 \\
1 & 1
\end{array}\right]=i P_{2} \\
& A([1,2],[2,3])=\left[\begin{array}{cc}
1 & 0 \\
2 i & 1
\end{array}\right]=P_{1}
\end{aligned}
$$

and their cofactors are

$$
\begin{gathered}
\AA([1,2],[1,2])=\left\{\begin{array}{rr}
i P_{n-2} & , n \equiv 0(\bmod 4) \\
-P_{n-2} & , n \equiv 1(\bmod 4) \\
-i P_{n-2} & , n \equiv 2(\bmod 4) \\
P_{n-2} & , n \equiv 3(\bmod 4)
\end{array}\right. \\
\AA([1,2],[1,3])=\left\{\begin{array}{rr}
-P_{n-3} & , n \equiv 0(\bmod 4) \\
-i P_{n-3} & , n \equiv 1(\bmod 4) \\
P_{n-3} & , n \equiv 2(\bmod 4) \\
i P_{n-3} & , n \equiv 3(\bmod 4)
\end{array}\right) \\
\AA([1,2],[2,3])=0 .
\end{gathered}
$$

By using the Laplace expansion the determinant of the matrix $N(n-1)$ is

$$
\operatorname{det}(N(n-1))=\left\{\begin{array}{rr}
-i P_{3} P_{n-2}-i P_{2} P_{n-3} & , n \equiv 0(\bmod 4) \\
P_{3} P_{n-2}+P_{2} P_{n-3} & , n \equiv 1(\bmod 4) \\
i P_{3} P_{n-2}+i P_{2} P_{n-3} & , n \equiv 2(\bmod 4) \\
-P_{3} P_{n-2}-P_{2} P_{n-3} & , n \equiv 3(\bmod 4)
\end{array} .\right.
$$


From (11) and(7), we obtain

$$
P_{n}=P_{3} P_{n-2}+P_{2} P_{n-3} .
$$

If the first three rows of the matrix $N(n-1)$ are chosen, there are only four $3 \times 3$ submatrices of the matrix $N(n-1)$ whose determinants are nonzero:

$$
\begin{aligned}
& A([1,2,3],[1,2,3])=\left[\begin{array}{ccc}
2 i & 1 & 0 \\
1 & 2 i & 1 \\
0 & 1 & 2 i
\end{array}\right]=-i P_{4} \\
& A([1,2,3],[1,2,4])=\left[\begin{array}{ccc}
2 i & 1 & 0 \\
1 & 2 i & 0 \\
0 & 1 & 1
\end{array}\right]=-P_{3} \\
& A([1,2,3],[1,3,4])=\left[\begin{array}{ccc}
2 i & 0 & 0 \\
1 & 1 & 0 \\
0 & 2 i & 1
\end{array}\right]=i P_{2} \\
& A([1,2,3],[2,3,4])=\left[\begin{array}{ccc}
1 & 0 & 0 \\
2 i & 1 & 0 \\
1 & 2 i & 1
\end{array}\right]=P_{1}
\end{aligned}
$$

and their cofactors are

$$
\begin{gathered}
\AA([1,2,3],[1,2,3])=\left\{\begin{array}{rr}
P_{n-3} & , n \equiv 0(\bmod 4) \\
i P_{n-3} & , n \equiv 1(\bmod 4) \\
-P_{n-3} & , n \equiv 2(\bmod 4) \\
-i P_{n-3} & , n \equiv 3(\bmod 4)
\end{array}\right. \\
\AA([1,2,3],[1,2,4])=\left\{\begin{array}{rr}
i P_{n-4} & , n \equiv 0(\bmod 4) \\
-P_{n-4} & , n \equiv 1(\bmod 4) \\
-i P_{n-4} & , n \equiv 2(\bmod 4) \\
P_{n-4} & , n \equiv 3(\bmod 4)
\end{array}\right) \\
\AA([1,2,3],[1,3,4])=0 \\
\AA([1,2,3],[2,3,4])=0
\end{gathered}
$$

By using the Laplace expansion the determinant of the matrix $N(n-1)$ is evaluated as follows:

$$
\operatorname{det}(N(n-1))=\left[\begin{array}{rr}
-i P_{4} P_{n-3}-i P_{3} P_{n-4} & , n \equiv 0(\bmod 4) \\
P_{4} P_{n-3}+P_{3} P_{n-4} & , n \equiv 1(\bmod 4) \\
i P_{4} P_{n-3}+i P_{3} P_{n-4} & , n \equiv 2(\bmod 4) \\
-P_{4} P_{n-3}-P_{3} P_{n-4} & , n \equiv 3(\bmod 4)
\end{array} .\right.
$$

From (11) and(9), we have

$$
P_{n}=P_{4} P_{n-3}+P_{3} P_{n-4} .
$$

The remainig identities in (4) can be shown similarly. 
Now, we give another proof of following Pell identity:

$$
P_{2 n}=P_{n}\left(P_{n+1}+P_{n-1}\right) .
$$

If we choose the first $(n-1)$ rows of the matrix $N(2 n-1)$, there are only $n$ the $(n-1) \times(n-1)$ submatrices of the matrix $N(2 n-1)$ whose determinants are nonzero but only the cofactors of two of them are nonzero. i.e.

$$
\begin{aligned}
& A([1,2, \ldots, n-1],[1,2, \ldots, n-2, n-1])=\left\{\begin{aligned}
-i P_{n} & , n \equiv 0(\bmod 4) \\
P_{n} & , n \equiv 1(\bmod 4) \\
i P_{n} & , n \equiv 2(\bmod 4) \\
-P_{n} & , n \equiv 3(\bmod 4)
\end{aligned}\right. \\
& A([1,2, \ldots, n-1],[1,2, \ldots, n-2, n])=\left\{\begin{aligned}
-P_{n-1} & , n \equiv 0(\bmod 4) \\
-i P_{n-1} & , n \equiv 1(\bmod 4) \\
P_{n-1} & , n \equiv 2(\bmod 4) \\
i P_{n-1} & , n \equiv 3(\bmod 4)
\end{aligned}\right.
\end{aligned}
$$

and their cofactors are

$$
\begin{gathered}
\AA([1,2, \ldots, n-1],[1,2, \ldots, n-2, n-1])=\left\{\begin{aligned}
P_{n+1} & , n \equiv 0(\bmod 4) \\
i P_{n+1} & , n \equiv 1(\bmod 4) \\
-P_{n+1} & , n \equiv 2(\bmod 4) \\
-i P_{n+1} & , n \equiv 3(\bmod 4)
\end{aligned}\right. \\
\AA([1,2, \ldots, n-1],[1,2, \ldots, n-2, n])=\left\{\begin{aligned}
i P_{n} & , n \equiv 0(\bmod 4) \\
-P_{n} & , n \equiv 1(\bmod 4) \\
-i P_{n} & , n \equiv 2(\bmod 4) \\
P_{n} & , n \equiv 3(\bmod 4)
\end{aligned}\right.
\end{gathered}
$$

From Laplace expansion the determinant of the matrix $N(2 n-1)$ is

$$
\operatorname{det}(N(2 n-1))=\left\{\begin{array}{rr}
-i P_{n}\left(P_{n+1}+P_{n-1}\right) & , n \equiv 0,2(\bmod 4) \\
i P_{n}\left(P_{n+1}+P_{n-1}\right) & , n \equiv 1,3(\bmod 4)
\end{array} .\right.
$$

From (11) and (12) we have

$$
P_{2 n}=P_{n}\left(P_{n+1}+P_{n-1}\right) .
$$

Thus the proof is completed.

\section{References}

[1] M. Yasar, H. Kiyak, D. Bozkurt, Complex Factorization Formulas for Fibonacci and Pell Numbers, The First International Conference on Mathematics and Statistics, American Sarjah University, U.A.E, March, 18$21,2010$.

[2] J. Feng, Fibonacci identities via the determinant of tridiagonal matrix, Applied Mathematics and Computation, 217, 5978-5981, 2011. 
[3] N. D. Cahill, J. R. D'Errico, J. Spence, Complex Factorizations of the Fibonacci and Lucas Numbers, Fibonacci Quarterly, 41(1), 13-19, 2003.

[4] M. Akbulak, F. Yilmaz, D. Bozkurt, Complex Factorization of the Fibonacci Numbers by Anti-tridiagonal Matrix Method, The 3rd Int. Workshop on Matrix Analysis and Appl. Zhejiang Forestry University, Hangzhou/Lin'An, China, July, 9-13, 2009. 